\title{
BROKEN SYMMETRIES AND THE MASSES OF GAUGE BOSONS
}

\author{
Peter W. Higgs \\ Tait Institute of Mathematical Physics, University of Edinburgh, Edinburgh, Scotland
}

(Received 31 August 1964)

In a recent note ${ }^{1}$ it was shown that the Goldstone theorem, ${ }^{2}$ that Lorentz-covariant field theories in which spontaneous breakdown of symmetry under an internal Lie group occurs contain zero-mass particles, fails if and only if the conserved currents associated with the internal group are coupled to gauge fields. The purpose of the present note is to report that, as a consequence of this coupling, the spin-one quanta of some of the gauge fields acquire mass; the longitudinal degrees of freedom of these particles (which would be absent if their mass were zero) go over into the Goldstone bosons when the coupling tends to zero. This phenomenon is just the relativistic analog of the plasmon phenomenon to which Anderson ${ }^{3}$ has drawn attention: that the scalar zero-mass excitations of a superconducting neutral Fermi gas become longitudinal plasmon modes of finite mass when the gas is charged.

The simplest theory which exhibits this behavior is a gauge-invariant version of a model used by Goldstone ${ }^{2}$ himself: Two real ${ }^{4}$ scalar fields $\varphi_{1}, \varphi_{2}$ and a real vector field $A_{\mu}$ interact through the Lagrangian density

$$
\begin{aligned}
L=-\frac{1}{2}\left(\nabla \varphi_{1}\right)^{2} & -\frac{1}{2}\left(\nabla \varphi_{2}\right)^{2} \\
& -V\left(\varphi_{1}^{2}+\varphi_{2}^{2}\right)-\frac{1}{4} F_{\mu \nu} F^{\mu \nu},
\end{aligned}
$$

where

$$
\begin{gathered}
\nabla_{\mu} \varphi_{1}=\partial_{\mu} \varphi_{1}-e A_{\mu} \varphi_{2}, \\
\nabla_{\mu} \varphi_{2}=\partial_{\mu} \varphi_{2}+e A_{\mu} \varphi_{1}, \\
F_{\mu \nu}=\partial_{\mu} A_{\nu}-\partial_{\nu} A_{\mu},
\end{gathered}
$$

$e$ is a dimensionless coupling constant, and the metric is taken as -+++ . $L$ is invariant under simultaneous gauge transformations of the first kind on $\varphi_{1} \pm i \varphi_{2}$ and of the second kind on $A_{\mu}$. Let us suppose that $V^{\prime}\left(\varphi_{0}^{2}\right)=0, V^{\prime \prime}\left(\varphi_{0}^{2}\right)>0$; then spontaneous breakdown of $U(1)$ symmetry occurs. Consider the equations [derived from (1) by treating $\Delta \varphi_{1}, \Delta \varphi_{2}$, and $A_{\mu}$ as small quantities] governing the propagation of small oscillations about the "vacuum" solution $\varphi_{1}(x)=0, \varphi_{2}(x)=\varphi_{0}$ :

$$
\begin{gathered}
\partial^{\mu}\left\{\partial_{\mu}\left(\Delta \varphi_{1}\right)-e \varphi_{0} A_{\mu}\right\}=0, \\
\left\{\partial^{2}-4 \varphi_{0}^{2} V^{\prime \prime}\left(\varphi_{0}^{2}\right)\right\}\left(\Delta \varphi_{2}\right)=0, \\
\partial_{\nu} F^{\mu \nu}=e \varphi_{0}\left\{\partial^{\mu}\left(\Delta_{1}\right)-e \varphi_{0} A_{\mu}\right\} .
\end{gathered}
$$

Equation (2b) describes waves whose quanta have (bare) mass $2 \varphi_{0}\left\{V^{\prime \prime}\left(\varphi_{0}^{2}\right)\right\}^{1 / 2}$; Eqs. (2a) and (2c) may be transformed, by the introduction of new variables

$$
\begin{gathered}
B_{\mu}=A_{\mu}-\left(e \varphi_{0}\right)^{-1} \partial_{\mu}\left(\Delta \varphi_{1}\right), \\
G_{\mu \nu}=\partial_{\mu} B_{\nu}-\partial_{\nu} B_{\mu}=F_{\mu \nu},
\end{gathered}
$$

into the form

$$
\partial_{\mu} B^{\mu}=0, \quad \partial_{\nu} G^{\mu \nu}+e^{2} \varphi_{0}^{2} B^{\mu}=0 .
$$

Equation (4) describes vector waves whose quanta have (bare) mass $e \varphi_{0}$. In the absence of the gauge field coupling $(e=0)$ the situation is quite different: Equations (2a) and (2c) describe zero-mass scalar and vector bosons, respectively. In passing, we note that the right-hand side of $(2 c)$ is just the linear approximation to the conserved current: It is linear in the vector potential, gauge invariance being maintained by the presence of the gradient ter . $^{5}$

When one considers theoretical models in which spontaneous breakdown of symmetry under a semisimple group occurs, one encounters a variety of possible situations corresponding to the various distinct irreducible representations to which the scalar fields may belong; the gauge field always belongs to the adjoint representation. ${ }^{6}$ The model of the most immediate inter est is that in which the scalar fields form an octet under SU(3): Here one finds the possibility of two nonvanishing vacuum expectation values, which may be chosen to be the two $Y=0$, $I_{3}=0$ members of the octet. ${ }^{7}$ There are two massive scalar bosons with just these quantum numbers; the remaining six components of the scalar octet combine with the corresponding components of the gauge-field octet to describe 
massive vector bosons. There are two $I=\frac{1}{2}$ vector doublets, degenerate in mass between $Y= \pm 1$ but with an electromagnetic mass splitting between $I_{3}= \pm \frac{1}{2}$, and the $I_{3}= \pm 1$ components of a $Y=0, I=1$ triplet whose mass is entirely electromagnetic. The two $Y=0, I=0$ gauge fields remain massless: This is associated with the residual unbroken symmetry under the Abelian group generated by $Y$ and $I_{3}$. It may be expected that when a further mechanism (presumably related to the weak interactions) is introduced in order to break $Y$ conservation, one of these gauge fields will acquire mass, leaving the photon as the only massless vector particle. A detailed discussion of these questions will be presented elsewhere.

It is worth noting that an essential feature of the type of theory which has been described in this note is the prediction of incomplete multiplets of scalar and vector bosons. ${ }^{8}$ It is to be expected that this feature will appear also in theories in which the symmetry-breaking scalar fields are not elementary dynamic variables but bilinear combinations of Fermi fields. ${ }^{9}$

\footnotetext{
${ }^{1} \mathrm{P} . \mathrm{W}$. Higgs, to be published.

${ }^{2} \mathrm{~J}$. Goldstone, Nuovo Cimento 19, 154 (1961); J. Goldstone, A. Salam, and S. Weinberg, Phys. Rev . 127,965 (1962).

${ }^{3}$ P. W. Anderson, Phys. Rev $\underline{130,} 439$ (1963).
}

In the present note the model is discussed mainly in classical terms; nothing is proved about the quantized theory. It should be understood, therefore, that the conclusions which are presented concerning the masses of particles are conjectures based on the quantization of linearized classical field equations. However, essentially the same conclusions have been reached independently by F. Englert and R. Brout, Phys. Rev . Letters 13, 321 (1964): These authors discuss the same model quantum mechanically in lowest order perturbation theory about the self-consistent vacuum.

${ }^{5}$ In the theory of superconductivity such a term arises from collective excitations of the Fermi gas.

${ }^{6}$ See, for example, S. L. Glashow and M. Gell-Mann, Ann. Phys. (N.Y.) 15, 437 (1961).

${ }^{7}$ These are just the parameters which, if the scalar octet interacts with baryons and mesons, lead to the Gell-Mann-Okubo and electromagnetic mass splittings: See S. Coleman and S. L. Glashow, Phys. Rev。134, B671 (1964).

${ }^{8}$ Tentative proposals that incomplete SU(3) octets of scalar particles exist have been made by a number of people. Such a rôle, as an isolated $Y= \pm 1, I=\frac{1}{2}$ state, was proposed for the $\kappa$ meson $(725 \mathrm{MeV})$ by Y. Nambu and J. J.Sakurai, Phys. Rev. Letters 11, 42 (1963). More recently the possibility that the $\sigma$ meson $(385$ $\mathrm{MeV}$ ) may be the $Y=I=0$ member of an incomplete octet has been considered by L. M. Brown, Phys. Rev . Letters 13,42 (1964).

${ }^{9}$ In the theory of superconductivity the scalar fields are associated with fermion pairs; the doubly charged excitation responsible for the quantization of magnetic flux is then the surviving member of a $U(1)$ doublet.

\title{
SPLIT TING OF THE 70-PLET OF SU(6)
}

\author{
Mirza A. Baqi Bég \\ The Rockefeller Institute, New York, New York \\ and \\ Virendra Singh* \\ Institute for Advanced Study, Princeton, New Jersey \\ (Received 18 September 1964)
}

1. In a previous note,$^{1}$ hereafter called $I$, we proposed an expression for the mass operator responsible for lifting the degeneracies of spinunitary spin supermultiplets [Eq. (31)-I]. The purpose of the present note is to apply this expression to the 70-dimensional representation of SU(6).

The importance of the 70-dimensional representation has already been underlined by Pais. ${ }^{2}$ Since

$$
\underline{35} \otimes \underline{56}=\underline{56} \oplus \underline{70} \oplus \underline{700} \oplus \underline{1134},
$$

it follows that 70 is the natural candidate for accommodating the higher meson-baryon reso- nances. Furthermore, since the $\mathrm{SU}(3) \otimes \mathrm{SU}(2)$ content is

$$
\underline{70}=(\underline{1}, \underline{2})+(\underline{8}, \underline{2})+(\underline{10}, \underline{2})+(\underline{8}, \underline{4})
$$

we may assume that partial occupancy of the 70 representation has already been established through the so-called $\gamma$ octet $^{2}\left(\frac{3}{2}\right)^{-}$. Recent experiments appear to indicate that some $\left(\frac{1}{2}\right)^{-}$ states may also be at hand. ${ }^{3}$ With six masses at one's disposal, our formulas can predict the masses of all the other occupants of 70 and also provide a consistency check on the input. Our discussion of the 70 representation thus appears to be of immediate physical interest. 